\title{
The Understanding of Major Depressive Disorder: a Multidimensional Analysis from Evolution to Cognitive Neuroscience
}

\author{
Banghan Xiao* \\ Saint Stephen's Episcopal School \\ *Corresponding author e-mail: Ericxiao2001@gmail.com
}

Keywords: Mental disorder, Norepinephrine, Dopamine, Major depressive disorder, Bipolar disorder, Schizophrenia, Darwinian medicine, Grey matter

\begin{abstract}
From the $19^{\text {th }}$ to $20^{\text {th }}$ century, many different approaches to mental disorder topic have been published to promote a more informative and comprehensive understanding of Major Depressive Disorder (MDD). However, previous studies on MDD had gone in-depth on a specific area, but failed to combine the understanding of MDD in other areas. The areas include evolutionary, genetic, hormones, brain structures, brain function, and early childhood trauma. Specific inferences are drawn as to how and in what ways these areas approach on mental disorder help to solve the mystery of major depressive disorder. This article is focusing on a variety of perspectives of Major Depressive Disorder (MDD) that mentioned above and helps to educate readers who are unfamiliar with this type of mental disorder. With the joint efforts of scholars in various fields, the mental health community will have huge success in defending mental disorder.
\end{abstract}

\section{Introduction}

Understanding the cause of mental disorders has traditionally, and even currently, been a complex and difficult challenge, such as Major Depressive Disorder, the mental disorders known as human medical misery for the past decades. Patients with Major Depressive Disorder (MDD) is characterized by their depressed emotions all the time. What bound with negative emotions is that individual is easy to be left alone, cut out from social connections, and develop unhealthy cognitive functions. According to the World Health Organization, by the year 2025, this mental disease will probably become the number two disability in the world. The various classifications and perspectives from different areas underwent many revisions but still can't guarantee to fix the mental disorder. Unlike physical diseases with very specific causes that can usually be identified and treated, or a bacteria disease that doctor simply use antibiotics to deal with, biological causes of mental disorders have been difficult to validate. To totally understand what can cause Major Depressive Disorder, it is necessary to further explore this issue from a variety of perspectives. From the evolutionary and genetics perspective, there are mutations selection and balancing selection prove the likelihood of group of population caught mental disorder. For the brain structure and brain function perspective, there are lots of studies using FMRI, EEG, PET, and CAT scans to discover the significant abnormalities inside the brain with patients having mental disorder. Besides that, there are other factors such as childhood trauma and etc. The primary focus of these studies has been on the most well-known mental disorder, i.e., major depressive disorder. To better prove the significance of a different perspective on MDD, others will be used as a comparison to it. The usefulness of these findings along with some reservations is detailed. The better understanding on the variety of the perspectives of mental disorder will help to make a more appropriate judgment on people's mental health. Because back in early $18^{\text {th }}-19^{\text {th }}$ centuries, the mental disorder has been a myth to people, all of the explanations to the patient's bizarre behavior are mostly theoretical, not practical. Even the cruelest practical brain surgery-lobotomy didn't even appear before the mid-19th century. 


\section{Biological Standpoint}

From the Biological standpoint of mental disorder, scholars and researchers have discovered lots of biological determinants that can cause mental disorders. For example, from the Evolution biology perspective, the Darwin medicine theory explained how human body was built for certain things and how diseases exist, and so on [1]. Based on the idea of Darwin medicine, inside of human gene pool, there are certain genetic alleles that are inside of human body help them stay away from the rare mental illness [2]. Interestingly, the mutations selection is exactly the answer to previous-points. The mutation selection proved the likelihood which group of population have higher risk to caught mental disorder [3]. However, mutation selection is not the only way to explain the likelihood of having server mental disorder. The idea of balancing selection also explains the genetic alleles associated with mental disorder [4]. Finally, there are hormones and neurotransmitter, the last two-piece puzzles in these complex equations. For example, there are norepinephrine, dopamine, and serotonin. These three factors are usually the main causes of Major Depressive Disorder [5].

\subsection{Evolutionary genes increased the risk of mental disorder}

As a primate, from the perspective of evolution, human as a species has changed a lot, and behind all of this, there is one gene pool can change over time as human passed down their generations. In the article, The Evolutionary Persistence of Genes That Increase Mental Disorders Risk, the author Keller suggested during the process of natural selection, some of the generic alleles changes during this process, and this negligible can allele be the key to understand what increases the risk of server mental disorders [6]. The evidence is simple and self-explanatory, the Darwin medicine takes Evolution of natural selection into account, and it explains human's certain behavior and deep down what their genes are built for. For example, anxiety can be awful rather than useful if people trigger them at the wrong time. This kind of nervous system, known as Fight or flight response, was built hundreds and thousands of years ago when human ancestor was trying to run away from the predator [7]. These are the traits imprinted in human's gene pool and passed down to thousands of generations after. How does this have anything to do with mental disorders? To demonstrate this phenomenon, it is supposed that there are some alleles passed down from human ancestors mean protecting people from mental illness, however during this process of evolution, some alleles removed or disappeared due to the error in DNA replication. This situation will make that person more vulnerable and susceptible to that mental disorder. On the recent conference on mental disorder, group of doctors have pointed out there are certain genes that helps to develop mental disorder, include ADHD, bipolar disorder, schizophrenia, autism spectrum disorder and major depression [8]. The way how it works is that these genes shared the same biological pathway in human brain, and when the brain cell hits the receptors, it can do all sorts of things.

\subsection{Hormone and neurotransmitter}

Hormone and neurotransmitter have always been the key components in mental disorder. For example, the earliest well-known explanation for Major Depressive Disorder from the biological perspective is the norepinephrine hypothesis of depression. The theory was suggested around the 1960s, when the first antidepressant just came out. The idea behind it suggests that norepinephrine is one major player in Major Depressive Disorder. And what the Antidepressant does is that it doesn't allow the enzyme to break down norepinephrine. Since the norepinephrine doesn't disappear, it will just keep interacting with the receptor; then, the person feels better and depression goes away [9]. So what exactly do norepinephrine do?

On the mice experiment, the results reflect the fact that when the neuron is stimulated, the mice can be happy [10]. And the conclusion is that the norepinephrine is pretty useful in this pleasure pathway. However, when the dopamine was brought up, suddenly norepinephrine has lost its charm. Not just because Dopamine is the most abundant catecholamine neurotransmitter in the brain, dopamine is also considered as a neurotransmitter regulating various physiological functions 
of the central nervous system. Most importantly, doctors notice that dopamine has something to do with cocaine. That's intriguing, because first thing it comes to people mind is the pleasure drugs brings to them, and that's probably why people do them. The truth is that dopamine have to do with rewards driven experience, every day our essential activities are reinforced by human brain system, amount pleasure that people received from activities is directly related to the dopamine level [11]. By this meaning, the importance of dopamine can be clearly understood: low level of dopamine pretty much implies the person is unhappy.

\section{Neural mechanisms}

Neural mechanisms describe the group of brain regions and the function of each part. Throughout the year, a variety of new technologies have been applied to the studies done on this topic, such as fMRI, MRI, and other neuroimaging techniques. And those studies are the crucial part to understand the link between neural mechanisms and mental disorder. In fact, in the early 70s, the technique of brain structure imaging was widely used [12]. Everyone was trying to push the boundaries of human's understanding on mental disorders from the neural mechanism perspective. That's very fascinating, and with the technology now, scientists are able to use the brain structure imaging to find the difference of structures between a normal person and the person with mental disorders. Additionally, scientists are able to use the fMRI machine to perform all sorts of studies on mental disorder people and understand their brain function [13]. For example, when scientists performed the brain scan on the patient with PTSD, they noticed patients amygdala is over activities [14]. For the brain structural differences, there is a very noticeable difference on the size of hippocampus between a normal person and the person with schizophrenia [15].

\subsection{Brain structure}

Bain structure, the term speaks for itself. Inside the brain, there are three main parts, cerebrum, cerebellum, and brain stem [16]. The one of these three most closely related to MDD is probably cerebrum. How so? In fact, Cerebrum is known as the largest part of the brain. It covers the hippocampus, prefrontal cortex, basal ganglia, and etc. The majority of brain regions covers have a lot to do with depression. Supporting this view are the findings that indicates there is a significant deficit grey matter volume on the prefrontal cortex, anterior circulates cortex, and occipital gyrus [17]. The other evidence was based on MacLean theory on Triun brain. More accurately, patients with depression will have significant decreased on hippocampal volumes, amygdala core volume, and etc. The study on patients with major depressive disorder under brain scans have never been consistent, but scientists always finds out results of those studies shows overlapping brain regions. And the common result of those research can be seen as a significant answer that contributes to the topic of brain structure [18].

Not just MDD, some other mental disorders share the same psychiatric symptoms under the brain scan [19]. For example, Bipolar disorder shares some similarities with MDD. Studies on patients with Bipolar I disorder showed an increase in volume on the left temporal lobe, right Putamen, and right lateral ventricle. Besides these three, there are no more significance on brain regions between patients with Bipolar disorder and completely healthy individuals. However, when putting lithium as a factor to these equations, it will be a completely different story. In that case, patients with Biplor I disorder will have increased hippocampal and amygdala volumes, compared to the healthy individuals treated with lithium [20]. In conclusion, both mental disorders (Bipolar disorder and major depressive disorder) shared the abnormality on brain structures, specifically on the hippocampus and temporal lobe. However, speaking from a rigorous perspective, this statement is not one hundred percent correct. Because even they do shares commonality, MDD patients show decrease volume in several brain regions that mentioned above. Whereas Bipolar I disorder patient shows abnormalities in exactly same brain region, except it's increase volume. It is possible that both mental disorders are a stress-related disease, so it makes sense that they have a common brain region abnormality. However, MDD is dealing with depress emotions, patients with this type of disorder tend to feel worthless, lose the passion for the activities they once enjoyed. Whereas the 
patient with Bipolar disorder tends to have extreme emotional state with inconsistent emotion. For example, the person can feel like on top of the world in the first ten minutes, after that he starts to for no reason. (That's the exaggeration of how patients have drastic emotions shift)

\subsection{Brain function}

Behind all the nonsense behavior, brain function can always provide with some insight. And scientists find that all sorts of parts of the brain have their own unique function. For example, amygdala is most known for how it regulates our emotions, and hippocampus is known for how it helps to store long term memories. In fact, in the early 19s, German anatomist Brodmann brought the idea that divided the cerebral cortex into a series of anatomical regions based on cell structure. This idea had a huge impact on the later day brain scan report, and scientists can easily link certain parts activations with person's behavior.

Enough with the introduction of the Brodmann area system, dive back in MDD. On the functional studies done on MDD, researcher always found increased activation BA25, 28 (limbic regions) [21]. For those who don't know, the limbic system's function is seen as central emotions that trigger people's best and worst behavior. Nowadays, a variety of research had uncovered its structure (Amygdala, hippocampus, spectrum, habenula, and mammillary bodies). Most importantly, when it comes to emotion, there are no center area responsible for people's emotions [22]. And that's why mental disorders like depression are so complicated. The whole thing is like a complex. If one thing goes wrong, the whole thing is collapse.

One thing neuroscientist in clinical practice picked up a lot is that amygdala appears in a variety of mental disorders. So, what exactly does amygdala do? In the past, lots of research studies that done on the amygdala showed that the amygdala was highly associated with aggression. Experiment like stimuli the amygdala shows can provide person rage and caused aggression [23]. The patient with MDD have increased activation on amygdala can be the reason why patient commit suicide, since suicide can be seen as self-aggression.

Lastly, there is the hippocampus, the parts of the brain have everything do with memory and emotion. To support this definition, there is a famous case study, known as the HM case. Long story short, on September $1^{\text {st }}$ Henry Molaison got his hippocampus removed from his brain due to his severe seizure he is suffering from. During that time the function of hippocampus was unknown. However, the seizure stops like a miracle. And what happened after was that HM cannot form new memory. Scientist later found out in order to have people's memory stored long term, they have to have their immediate sensory data transcribed by in the cortex, later it has to travel through the hippocampus. Since HM's hippocampus was removed, there is no way for him to store long term information [24]. Tie back to MDD, patient with MDD does have a variety of problems dealing with the hippocampus, such as poor memorization and confusion.

\section{Childhood trauma}

Childhood has always been a big part of a person's life. Human develop caused and effect sense mostly in childhood. Without much doubt, childhood trauma can be very horrified consider how it struck a person and twist their cognitions to a false response to certain things. In the 20th century, lots of criminals have diagnosed with a mental disorder, and one thing they shared in common is an awful childhood. Also, evolutionary biologists have proven that early hood parenting care has a direct relationship to kids' anxiety levels when they reach adolescence. So basically, if the parent spends more time with the kid, taking care of them, and when kids reach the adolescent, they tend to develop less anxiety by the surrounding environment. Whereas, if the parent just decides to be irresponsible, abandoning the kids in early ages, the kid can develop anxiety pretty easily, compared to the kids who have good parenting care in early ages [25]. In short, anxiety is one of the biggest factors in depression since MDD is considered as stress-related diseases.

However, sometimes childhood trauma is inevitable, no one ever knows how this happens. It's so sudden, and those things are like a scar on people's skin and it can never be erased. Moreover, the MDD caused by early childhood trauma is hard to treat it with antidepressants. More accurately, 
antidepressant is not as efficient as psychotherapy in this situation [26].

\section{Conclusion}

The current understanding of Major Depressive Disorder is remarkable, scientists from a variety of the areas are able to find a way to approach to this mental disorder. Surely, biological determinant has always been a huge part of MDD, especially the antidepressants that help the mental health community. However, when every area works on MDD put together, a human can put a huge success on MDD. Sometimes patients tend to have a preference for one specific area that helps their mental illnesses. In order to help those patients, it is necessary to let them understand different perspectives to this problem. Those perspectives include evolutionary, genetic, hormones, brain structures, brain function and etc. Lastly, it is expected that in the next decade, the mental health community will have huge success in defending mental disorder. The understanding of major depressive disorder will help to eliminate the possibility getting it.

\section{References}

[1] Keller, M. (2008). The Evolutionary Persistence of Genes That Increase Mental Disorders Risk. Current Directions in Psychological Science, 17(6), 395-399.

[2] Peter, Falkai, et al. "Forty Years of Structural Brain Imaging in Mental Disorders: Is It Clinically Useful or Not?” Dialogues in Clinical Neuroscience, Les Laboratoires Servier, Sept. 2018,

[3] Gillihan, Seth J. “Using Brain Scans to Diagnose Mental Disorders.” Psychology Today, Sussex Publishers, 19 May 2016, www.psychologytoday.com/us/blog/think-act-be/201605/using-brainscans-diagnose-mental-disorders.

[4] “Using Brain Scans to Diagnose Mental Illness.” The Recovery Village, 28 Jan. 2020, www.therecoveryvillage.com/mental-health/related/using-brain-scans-to-diagnose-mentalconditions/\#gref.

[5] Nemeroff, C., Heim, C., Thase, M., Klein, D., Rush, A., Schatzberg, A., . . Keller, M. (2003). Differential Responses to Psychotherapy versus Pharmacotherapy in Patients with Chronic Forms of Major Depression and Childhood Trauma. Proceedings of the National Academy of Sciences of the United States of America, 100(24), 14293-14296.

[6] Mansari, M., Guiard, B., Chernoloz, O., Ghanbari, R., Katz, N., \&amp; Blier, P. (2010, April 08). Relevance of Norepinephrine-Dopamine Interactions in the Treatment of Major Depressive Disorder.

[7] Nesse, R. (2001, May). How is Darwinian medicine useful? Retrieved June 12, 2020, from https://www.ncbi.nlm.nih.gov/pmc/articles/PMC1071402/

[8] University of Queensland. (2019, July 24). Genes underscore five psychiatric disorders. ScienceDaily. Retrieved June 12, 2020 from www.sciencedaily.com/releases/2019/07/

[9] Norepinephrine - ADHD, Depression \& Low Blood Pressure: Everyday Health. (2015, December 11). Retrieved from https://www.everydayhealth.com/norepinephrine/guide/

[10] Kringelbach, M. L., \& Berridge, K. C. (2010). The functional neuroanatomy of pleasure and happiness. Discovery medicine, 9(49), 579-587

[11] Rössger, K., Hamri, G., \& Fussenegger, M. (2013). Reward-based hypertension control by a synthetic brain-dopamine interface. Proceedings of the National Academy of Sciences of the United States of America, 110(45), 18150-18155. Retrieved June 14, 2020, from www.jstor.org/stable/23754725

[12] Jacobs, B. (2004). Depression: The Brain Finally Gets into the Act. Current Directions in 
Psychological Science, 13(3), 103-106. Retrieved June 15, 2020, from www.jstor.org/stable/20182923

[13] Zhang, F. F., Peng, W., Sweeney, J. A., Jia, Z. Y., \& Gong, Q. Y. (2018). Brain structure alterations in depression: Psychoradiological evidence. CNS neuroscience \& therapeutics, 24(11), 994-1003. https://doi.org/10.1111/cns.12835

[14] Pandya, M., Altinay, M., Malone, D. A., Jr, \& Anand, A. (2012). Where in the brain is depression?. Current psychiatry reports, 14(6), 634-642. https://doi.org/10.1007/s11920-012-03227

[15] Khatchaturian, Z. (n.d.). Brain Tour. Retrieved from https://www.alz.org/alzheimersdementia/what-is-alzheimers/brain_tour

[16] Sapolsky, R. M. (2018). Behave: The biology of humans at our best and worst. NY, NY, New York: Penguin Books.

[17] Sheline Y. I. (2011). Depression and the hippocampus: cause or effect?. Biological psychiatry, 70(4), 308-309. https://doi.org/10.1016/j.biopsych.2011.06.006

[18] Deborah, H. (2018, August 28). The Curious Case of Patient H.M. Retrieved June 25, 2020, from https://www.brainfacts.org/in-the-lab/tools-and-techniques/2018/the-curious-case-of-patienthm-082818

[19] Sapolsky, R. M. (1994). Why zebras don't get ulcers: A guide to stress, stress related diseases, and coping. New York City, New York: W.H. Freeman.

[20] Hallahan, B., Newell, J., Soares, J. C., Brambilla, P., Strakowski, S. M., Fleck, D. E., ... \& McIntosh, A. M. (2011). Structural magnetic resonance imaging in bipolar disorder: an international collaborative mega-analysis of individual adult patient data. Biological psychiatry, 69(4), 326-335.

[21] Gillath, O., Bunge, S. A., Shaver, P. R., Wendelken, C., \& Mikulincer, M. (2005). Attachmentstyle differences in the ability to suppress negative thoughts: exploring the neural correlates. Neuroimage, 28(4), 835-847.

[22] Gobe, M. (2010). Emotional branding: The new paradigm for connecting brands to people. Simon and Schuster.

[23] Ulrich, R. (1966). Pain as a cause of aggression. American Zoologist, 6(4), 643-662.

[24] Milner, B. (1970). Memory and the medial temporal regions of the brain. Biology of memory, 23, 31-59.

[25] Weiss, R. S. (1979). Growing up a little faster: The experience of growing up in a single-parent household. Journal of Social Issues, 35(4), 97-111.

[26] Spielmans, G. I., Berman, M. I., \& Usitalo, A. N. (2011). Psychotherapy versus secondgeneration antidepressants in the treatment of depression: a meta-analysis. The Journal of nervous and mental disease, 199(3), 142-149. 